УДК 621.9.06

Шелковой А. Н., Набока Е. В.

Национальный технический университет «Харьковский политехнический институт». Украина, г. Киев

\title{
ИНТЕГРАЦИЯ АВТОМАТИЗИРОВАННОГО МЕХАНОСБОРОЧНОГО ПРОИЗВОДСТВА ВЫСОКОТОЧНЫХ МАШИНОСТРОИТЕЛЬНЫХ ИЗДЕЛИЙ КАК АЛЬТЕРНАТИВА ИХ СЕЛЕКТИВНОЙ СБОРКИ
}

В статье проведен анализ условий сборки высокоточных изделий без подгонки и селективного отбора. Сформулированы исходные посылки механосборочного производства на основе интеграции производственных процессов. Выделены параметры управления оборудованием для сборки и обработки деталей. Приведена математииеская модель процесса виртуальной сборки изделия и механической обработки резанием деталей, входящих в него, позволяющая осуществлять сборку высокоточных изделий без подгонки и селективного отбора. [dx.doi.org/10.29010/087.4]

Ключевые слова: высокоточное изделие; виртуальная сборка; механическая обработка резанием; конструкторская размерная цепь; технологическая размерная цепь; управление обработкой; управление сборкой; имитационное моделирование; математическая модель.

Введение

Проблема изготовления высокоточных изделий (ВИ) (изделия, в состав которых входят не менее 50\% деталей, у которых хотя бы одна из поверхно- стей имеет точность собственных размеров либо точность их взаимного расположения на детали не ниже 8 квалитета) в условиях мелкосерийного автоматизированного производства заключается в поиске баланса между затратами на сборочный 
процесс и механическую обработку резанием деталей, из которых состоит изделие.

Процесс сборки изделия характеризуется []:

- структурой, т.е. набором переходов сборки деталей в подузлы, узлы и готовые изделия, объединяемые в сборочные операции и процессы сборки изделия, которая отражает как состав деталей, входящих в изделие, так и последовательность их соединения в процессе сборки);

- параметрами (временными, энергетическими, технико-экономическими), состоящими из параметров переходов, которые рассчитываются исходя из выполняемых технологических приемов и средств, используемых для этого, а так же из параметров операций и производственного процесс в целом, расчет которых ведется на основании временных параметров переходов и анализа того, в какой последовательности (последовательно, параллельно или параллельно-последовательно) они выполняются в рамках рассматриваемой структуры (операции, процесса сборки). Существенное влияние на параметры процесс сборки оказывает уровень его автоматизации на различных уровнях технологического процесса (переход, операция, процесс).

Целью сборочного процесса является создание изделия с заданными функциональными характеристиками в условиях ограничений на материальные, трудовые и экономические ресурсы. Исходя из этого, его можно разделить на основные операции и переходы, в рамках которых формируются свойства изделия на заданном множестве деталей, узлов и механизмов и вспомогательные - обеспечивающие нормальное функционирование основных операций и переходов.

Практика производства изделий показывает, что не всегда детали, соответствующие требованиям чертежа, могут быть использованы в изделии, так как в сочетании с другими деталями они иногда могут не обеспечить его собираемость. Поэтому в технологический процесс сборки изделия, в зависимости от вида изделия и программы ее выпуска, добавляют вспомогательные операции и переходы (селекцию, подгонку, регулировку, притирку, установку различного вида компенсаторов и т.д.), главной задачей которых является компенсация возникающих дополнительных погрешностей в ходе сборки. При этом широко применяется селекция деталей на основе конструкторских размерных цепей, в которых они участвуют своими размерами.

Если при создании узлов из деталей с нормальной точностью, их избыточность, для заданной партии изделий, не существенно увеличивает его себестоимость (особенно в условиях крупносерийного и массового производства), то при создании ВИ малыми партиями это приводи к существенному удорожанию изделия (до $30 \%$ от планируемой себестоимости).
Таким образом, задача разработки интегрированной технологии механосборочного производства ВИ, исключающую селекцию деталей и минимальную (по трудоемкости) подгонку и регулировку деталей в ВИ является актуальной для точного машиностроения, станкостроения и аэрокосмической промышленности, а так же других машиностроительных отраслей, в которых используются сложные, высокоточные изделия.

\section{Постановка задачи исследования}

Одним из основных условий создания автоматизированного производства ВИ без дополнительных затрат, связанных с подгонкой деталей в изделии, является наличие прямых и обратных информационных связей между системами управления сборочным, металлорежущим и контрольно-измерительным оборудованием с целью обеспечения $100 \%$ гарантии собираемости изделия из изготовленных деталей при минимальной длительности производственного цикла (рис. 1).

В качестве исходных данных для построения технологического процесса сборки изделия технолог использует либо данные сборочного чертежа, либо 3D - модель этого изделия, созданную в одной из CAD - систем (рис. 1).

При этом он ничего не знает о реальных размерах деталей, входящих в изделие, а оперирует их «идеальными» образами в виде номинальных размеров и максимально возможных, для данной сборки, отклонений от номинала. Предполагается, что детали, полученные в соответствии с их техническими требованиям и чертежами, будут иметь размеры, обеспечивающие собираемость изделия с учетом расчетных сборочных размерных цепей.

Но так бывает далеко не всегда. Приблизительно 20\% случаев сборки изделий из годных деталей их теоретические и реальные размерные цепи не совпадают, что приводит либо к браку, либо к необходимости селективного подбора деталей для конкретного узла [1].

При выпуске высокоточных изделий малыми партиями необходим другой подход к формированию, как технологии сборки изделия, так и технологий формообразования деталей (ТФД), из которых собирается узел (рис. 1).

Он заключается в том, что параллельно с формообразованием деталей реализуется виртуальный сборочный процесс изделия (ВСПИ), в котором могут присутствовать как реальные размеры уже изготовленных деталей, так и виртуальные размеры поверхностей деталей, которые предстоит еще создать (рис. 1, [3, 4]). ВСПИ должен помочь решить три задачи: 1) контролировать собираемость узла с учетом размеров поверхностей уже изготовленных деталей; 2) периодически позволять 


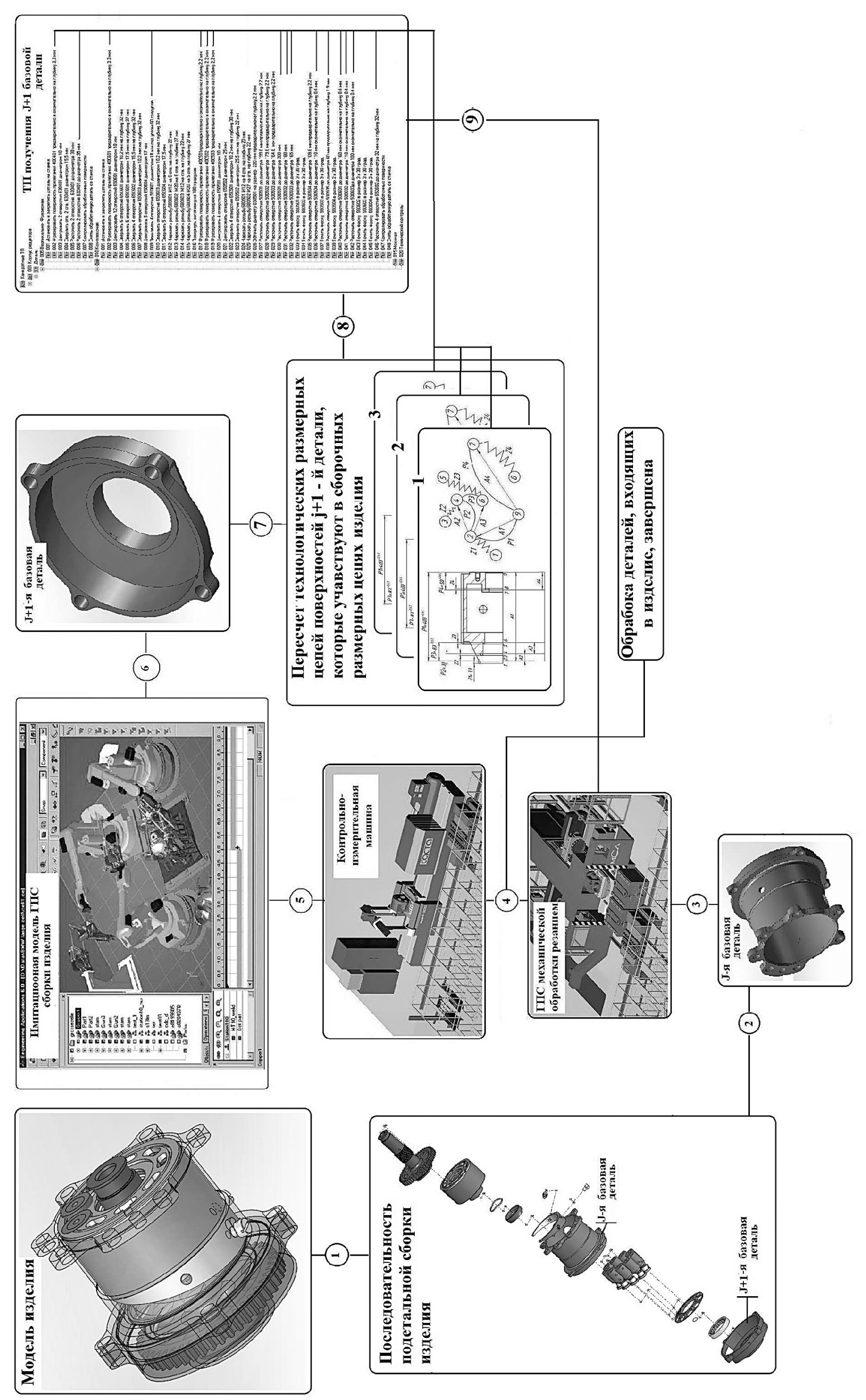


корректировать размерные цепи с учетом уже изготовленных деталей для размеров поверхностей деталей, которые предстоит изготовить; 3) передавать в очередной ТФД пересчитанные размеры в виде настроенных с целью корректировки управляющих программ обрабатывающих центров или станков с ЧПУ ([1, 5], рис. 1).

При кажущейся простоте такого подхода к формированию ТФД, в его реализации есть ряд трудностей:

- последовательность реальной сборки изделия ([1], рис. 1) должна строго совпадать с последовательностью ВСПИ ([2-4], рис. 1), от этого зависит, в какой последовательности можно изготавливать детали, входящие в узел так, чтобы уложиться в допуск на размер замыкающего звена сборочной цепи;

- в сборочные размерные цепи входят не все размер деталей, а только их часть; для формирования этих размеров в ТФД существую операции и переходы, которыми нужно управлять при наладке станка ([7-8], рис. 1);

- в отличие от существующих ТФД, для которых характерно формообразование деталей, входящих в один узел, параллельное без связи друг с другом, для ТФД с ВСПИ существует четкая иерархия изготовления деталей, в которой деталь, стоящая ниже в иерархии, не может быть изготовлена параллельно или раньше детали, стоящей выше нее;

- формирование иерархии деталей, подлежащих обработке и определение моментов времени, когда можно запускать ТФД ([2, 6], рис. 1) так же является непростой задачей, в которой сходятся как технологические, так и организационные проблемы повышения производительности обработки при сохранении 100\% гарантии собираемости узла.

- расчет конструкторских и технологических размерных цепей ([5, 7], рис. 1) превращается не в единовременный акт, а повторяется по мере формирования реальных размеров деталей, при этом возможна их корректировка в зависимости от результатов обработки в предыдущих ТФД.

\section{Математическая модель механосборочного процесса}

Для описания математической модели процесса производства ВИ дадим ряд определений, связанных с формированием иерархии деталей в узле:

Базовая деталь $(\boldsymbol{b} d)$ - деталь изделия $(d)$ либо участвующая в реализации его функций, либо обеспечивающая базирование других деталей на ней, либо выполняющая и то, и другое.

Характер взаимосвязей между базовыми деталями определяются на основе анализа формы контакта между их поверхностями в сборочном узле.
Крепежная деталь $(\boldsymbol{k} \boldsymbol{d})$ - деталь, в функции которой входит обеспечение силового замыкания, благодаря которому можно создать сборочную единицу (гайки, болты, заклепки и др.).

Сборочная единица (se) - часть изделия, созданная базовыми и крепежными деталями и обладающая конструктивной самодостаточностью, т.е. не разрушающаяся в процесс его произвольного перемещения в пространстве.

\section{Формализация процесса сборки изделия}

Сформулируем ряд исходных аксиом процесса сборки:

1. Если деталь $\left(d_{i}\right)$ совмещает в себе сразу две функции базирования $(b)$ на ней детали с индексом «n» и крепления $(k)$ набора деталей, то она считается базовой $(b)$ и справедливо утверждение (1)

$$
S V d_{i} \ni(b, k) \rightarrow\left(d_{i} \equiv b d_{i, n}\right),
$$

где: $S V d_{i}-$ свойство $i$-й детали; $\equiv-$ тождественность.

2. Крепежные детали, при сборке изделия, всегда устанавливают после базовых деталей $(\overline{1, N})$, формирующих конструкторскую размерную цепь $(K R C)$, утверждение (2)

$\underset{i=1}{\forall-1} b d_{i, j, \ldots, n-1} \exists k d_{i}\left[\begin{array}{l}\left(K R C \ni b d_{i, j, \ldots, n-1}\right) \cdots \rightarrow \\ \cdots \rightarrow\left(b d_{1,2} \prec b d_{2,3} \prec b d_{3, \ldots} \prec \ldots \prec\right. \\ \left.\prec \ldots \prec b d_{N-1, N} \prec b d_{N, 1} \prec k d_{1}\right)\end{array}\right] ;$

где: ঝ - знак последовательности установки деталей (узлов) в изделии;

$(\alpha) \rightarrow(\beta)-$ из утверждения « $\alpha \gg$ следует утверждение « $\beta$;

В выражении (2) индексы первой базовой детали и крепежной совпадают, что говорит о том, что крепежная деталь крепится на базовой детали.

3 . Если базовые детали $(i, j, k)$ устанавливаются в последовательности: $b d_{i, j} \prec b d_{j, k} ; b d_{j, k} \prec d_{k}$ и $d_{i} \odot d_{k}$, тогда $b d_{j, k} \prec d_{k}$, что фор-

мально можно записать в виде утверждения (3)

$\left(\left(b d_{i, j} \prec b d_{j, k}\right) \wedge\left(b d_{j, k} \prec b d_{k}\right) \wedge d_{i} \odot d_{k}\right) \rightarrow\left(b d_{i, k} \prec b d_{k, i}\right) .(3)$

В соответствии со схемой (рис. 1), на первом этапе сборки ВИ без селективного отбора определим последовательность сборки деталей в узлы. Чтобы определить порядок следования базовых деталей в изделии необходимо количественно оценить влияние последовательности их сборки на эффективность технологического процесса. Для этого сформулируем гипотезы, позволяющие выполнить такую оценку:

- В узле в качестве базовых деталей, как правило, используются детали, имеющие наибольшую мaccy; 
- В узле для базирования из множества, связанных между собой, деталей выбирают ту деталь, у которой максимальная суммарная площадь контакта поверхностей с другими деталями;

- В узле для базирования деталей из множества связанных между собой деталей выбирают ту деталь, у которой максимальное количество контактов с другими деталями в узле.

При определении базовой детали необходимо рассчитать значение комплексного критерия, базирующегося на приведенных выше гипотезах.

Одна такой расчет сопряжен с рядом трудностей:

- каждый из, перечисленных выше, параметров имеет свою природу формирования и размерность и поэтому не может быть использован напрямую;

- достоверно не известна степень влияния каждого из параметров на решение о принятии детали в качестве базовой;

- у перечисленных выше параметров отсутствуют корреляционные связи.

Исходя из выше сказанного, предлагается в качестве расчетных параметров выбора базовых деталей использовать безразмерные характеристики:

- для массовой оценки выбора $j$-й базовой детали рассчитать параметр $K M d_{j}$ по (4)

$$
K M d_{j}=\frac{m d_{j}}{\underset{i=1}{\forall}\left(\underset{k \ni N}{\exists} \operatorname{MAX}\left(m d_{k}\right)\right)},
$$

где: $m d_{i}-$ масса $j$-й детали; $\underset{i=1}{\forall} \underset{k \ni N}{\exists} M A X\left(m_{i}\right)$ - самая большая масса одной из деталей ( $k$-й детали), входящей в изделие, которое состоит из $N$ - деталей;

- для выбора $k$-й базовой детали по суммарной площади ее контакта с другими базовыми деталями рассчитать параметр $K S d_{k}$ по (5), [2]

$$
K S d_{j}=\frac{\underset{i=1}{\forall} \exists\left(b d_{k N}\left(\left(b d_{j} \odot b d_{k}\right)\right) \rightarrow S b d_{k}=\sum_{k=i}^{m_{j}} S b d_{j, k}\right)}{\left.{\underset{i=1}{\forall} \underset{j \ni N}{N} M A X\left(S b d_{j}\right)}_{\exists} M A\right)}
$$

где: $m_{j}$ - количество контактов $j$-й детали с другими деталями (исключая крепежные); $\underset{i=1}{\stackrel{N}{\exists} \exists N} M A X\left(k d_{k}\right)-$ самая большая площадь контакта одной из деталей, входящих в изделие из $N$ - деталей, с другими деталями ( $i$-й детали); $\odot-$ знак взаимодействия (контакта) двух деталей.

- для оценки выбора $j$-й базовой детали по количеству ее контактов с другими деталями рассчитать параметр $K K d_{j}$ по (6)

$$
K K d_{j}=\frac{k d_{j}}{\underset{i=1}{\forall}\left(\underset{k \ni N}{\exists} \operatorname{MAX}\left(k d_{k}\right)\right)},
$$

где: $k d_{j}-$ количество контактов $j$-й детали с другими деталями (исключая крепежные); $\underset{i=1}{\forall} \underset{k \ni N}{\exists} M A X\left(k d_{k}\right)$ - самая большая масса одной из деталей ( $k$-й детали), входящей в изделие, которое состоит из $N$ - деталей.

Переход к безразмерным параметрам позволяет осуществить комплексную оценку соответствия конкретной детали понятию «базовая» при ее включении в сборочную единицу.

Учитывая, что параметры не коррелируют между собой, будем рассчитывать значение комплексного коэффициента эффективности базовой детали $\left(K E d_{j}\right)$ по (7)

$$
K E d_{j}=K M d_{j}+K S d_{j}+K K d_{j} .
$$

С учетом того, что каждый из параметров, входящий в (7), изменяется в диапазоне от 0 до 1, значение коэффициента $K E d_{i}$ будет изменяться в диапазоне от 0 до 3. При этом правая граница (значение «3») будет отвечать максимальному соответствию $j-$ й детали понятию «базовая деталь» в рассматриваемом сборочном узле.

Тогда порядок соединения $j$-й детали с $i$-й деталью можно определить следующим образом (8)

$\left\{\begin{array}{l}\underset{\substack{j=1, n, j \\ j \neq i}}{\forall}\left(d_{i} \odot d_{j}\right) \wedge\left(K E d_{i}>K E d_{j}\right) \rightarrow\left(d_{i} \equiv b d\left(d_{j}\right) \text { или } b d_{i, j}\right) \\ b d_{i, j} \rightarrow\left(d_{i} \prec d_{j}\right) .\end{array}\right.$

Если базовая деталью $\left(b d_{i}\right)$ и крепежная деталь $\left(k d_{i}\right)$ формируют силовое замыкание базовых деталей с индексами $(j, k, \ldots, m)$ в одном из направлений их возможных перемещений, тогда можно утверждать, поверхности этих деталей могут создать конструкторскую размерную цепь независимой сборочной единицы $\left(s e_{i}\right)(9)$

$$
\begin{aligned}
& \left(\left(b d_{i, j} \prec b d_{j, k} \prec b d_{k, \ldots} \ldots \prec b d_{m, i}\right) \wedge\left(b d_{i, j} \prec k d_{i}\right)\right) \rightarrow \cdots \\
& \ldots \rightarrow\left(s e_{i} \equiv\left(b d_{i, j} \prec b d_{j, k} \prec b d_{k, \ldots} \ldots \prec b d_{m, i} \prec k d_{i}\right)\right) .
\end{aligned}
$$

Количество размерных цепей $\left(N_{R C}\right)$ определяется количеством крепежных деталей, устанавливаемых в узле $\left(N_{k d}\right)$.

$$
N_{R C}=f\left(N_{k d}\right) .
$$

6. Если несколько деталей устанавливаются на базовую деталь независимо друг от друга (отсутствие иерархии установки), тогда они образуют сложную систему размерных цепей, «укладывающихся» в единую размерную цепь по признаку расположения крепежных деталей. где «»- символ, обозначающий независимую установку деталей на базовую деталь.

Рассмотрим свертку деталей узла, имеющего конструктивную форму, математическое описание которой представлено (11) 


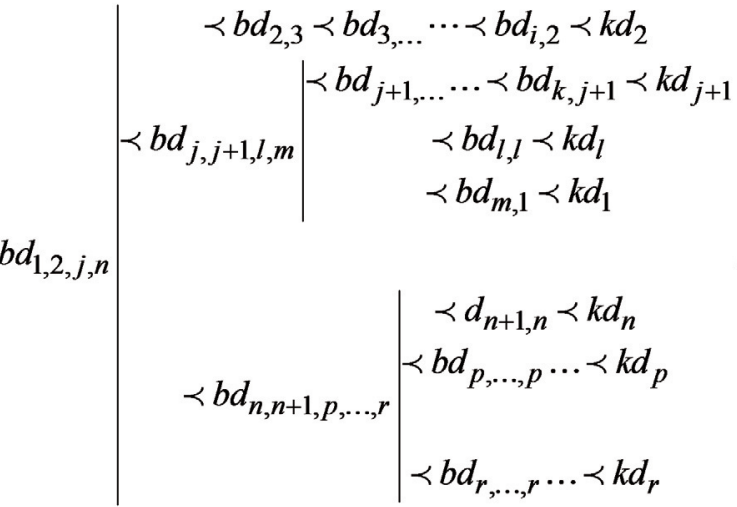

Анализ (11) показывает, что в узле можно выделить несколько подузлов:

- подузлы первого уровня, т.е. такие, в которые входят только детали, но в них нет более мелких подузлов, в нашем примере это подузлы $\left(s e_{2}, s e_{j+1}\right.$, $\left.s e_{l}, s e_{p}, s e_{r}\right)$, а их формальное описание представлено системой (12)

$$
\begin{aligned}
& s e_{2} \equiv b d_{2,3, \ldots i} \prec b d_{3, i} \prec \cdots \prec b d_{i, 2} \prec k d_{2}, \\
& s e_{j+1} \equiv b d_{j+1, \ldots, k} \prec \ldots \prec b d_{k, j+1} \prec k d_{j+1}, \\
& s e_{l} \equiv b d_{l, \ldots .} \ldots \prec k d_{l}, \\
& s e_{p} \equiv b d_{p, \ldots} \ldots \prec k d_{p}, \\
& s e_{r} \equiv b d_{r, \ldots} \ldots \prec k d_{r}
\end{aligned}
$$

Индекс подузла (se) указывает на номер базовой детали, на которой строится конструкторская цепь.

Тогда, с учетом (12), структура (11) преобразуется к виду (13)

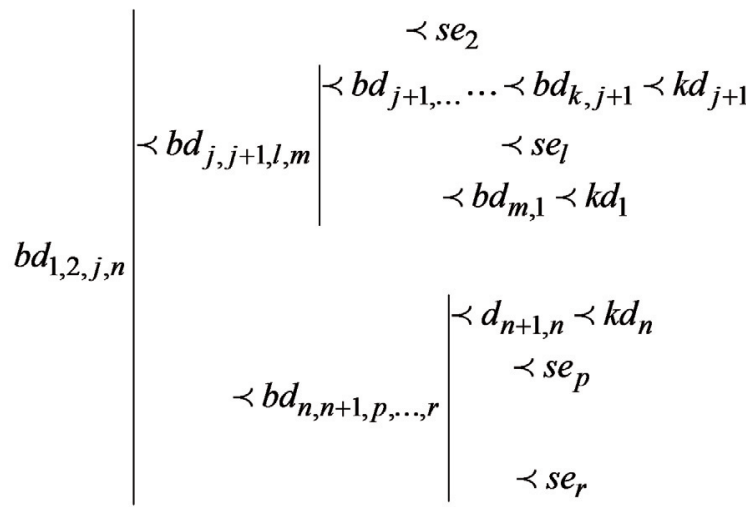

- подузлы второго уровня это такие подузлы, в которые, кроме отдельных деталей, могут входить более простые подузлы, в нашем примере их формальное описание представлено структурой (14)

$$
s e_{n} \equiv b d_{n, s e_{p}, s e_{r}, n+1} \mid \begin{gathered}
\prec b d_{n+1} \prec k d_{n} \\
\prec s e_{p} \\
\prec s e_{r}
\end{gathered} ;
$$

При сборке узла, как правило, подузлы (в нашем примере $s e_{p}$ и $s e_{r}$ ) устанавливаются на базовую деталь $\left(b d_{n}, s e_{p}, s e_{r},{ }_{n+1}\right)$ до того, как устанавливаются другие детали $\left(b d_{n+1}\right)$, формирующие силовое замыкание крепежной деталью $\left(k d_{n}\right)$ и размерную цепь. Последовательность установки подузлов $s e_{p}$ и $s e_{r}$ на базовую деталь, по аналогии с установкой деталей $d_{i}$ и $d_{j}$, определяется на основе их ранжирования по (15)

$$
\begin{aligned}
& \underset{\substack{j=1, n ; \\
j \neq i}}{\forall}\left[\left(s e_{i} \odot b d_{k, i, j}\right) \wedge\left(s e_{i} \odot b d_{k, i, j}\right)\right] \wedge\left(K E e_{i}>K E e_{j}\right) \rightarrow \\
& \rightarrow\left(b d_{k, i, j} \prec s e_{i} \prec s e_{j}\right) .
\end{aligned}
$$

Комплексный коэффициента эффективности последовательности установки подузла $K E e_{i}$ на базовую деталь определяется на основе расчета: его массы $\left(K M e_{j}\right)$; суммарной площади контакта подузла с базовой деталью, на которой он монтируется $\left(K S e_{j}\right)$; количества внешних связей подузла с другими подузлами и базовыми деталями $\left(K K e_{j}\right),(16)$

$$
K E e_{j}=K M e_{j}+K S e_{j}+K K e_{j}
$$

- тогда, если в (14) $\left(K E e_{r}>K E e_{p}\right)$, то структура (14) может быть записана в виде (17)

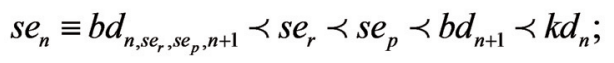

- тогда окончательную структуру изделия можно формально описать структурой (18)

$$
s e_{1} \equiv b d_{1, s e_{2}, s e_{n}, j, s e_{j+1}, s e_{l}, m} \mid \begin{gathered}
\prec s e_{2} \\
\prec s e_{j, s e_{j+1}, s e_{l}} \mid \begin{array}{c}
\prec s e_{l} \\
\prec d_{m} \prec k d_{1}
\end{array} . \\
\prec s e_{n}
\end{gathered}
$$

Приняв $\left(K E e_{2}>K E e_{n}\right)$ и $\left(K E e_{j+1}>K E e_{l}\right)$ структуpa (18) преобразуется к виду (19)

$$
\begin{aligned}
& s e_{1} \equiv b d_{1, s e_{2}, s e_{n}, j, s e_{j+1}, s e_{l}, m} \prec s e_{2} \prec s e_{n} \prec b d_{j, s e_{j+1}, s e_{l}} \prec \\
& \prec s e_{j+1} \prec s e_{l} \prec b d_{m} \prec k d_{1} .
\end{aligned}
$$

Формальное описание сборочной единицы в виде структурных объединений в подузль (19) позволяет, при моделировании механосборочного процесса, сформировать очередность формообразования деталей, входящих в изделие.

В соответствии со схемой (рис. 1), на втором этапе сборки ВИ без селективного отбора определим размерные характеристики поверхностей, входящих в конструкторские размерные цепи (КРЦ) деталей и подлежащих формообразованию. 
Допустим, детали $\left(d_{i}\right)$ принадлежит множество поверхностей $\left(P d_{i}\right)$, формирующих размерные цепи с другими деталями в узле. При этом определена последовательность ее установки в изделие $\left(s e_{1}\right)$.

Тогда поверхности $i$-й детали $\left(P d_{i}\right)$ можно разделить на поверхности, участвующие в формировании $k$-й КРЦ с деталями, которые устанавливаются в узел до $i$-й детали - $\left(P d_{k, i}^{-}\right)$и после ее установки $\left(P d_{k, i}^{+}\right),(20)$

$$
\left\{P d_{k, i}^{-}, P d_{k, i}^{+}\right\} \in P d_{k, i} .
$$

Известно, что при сборке изделия с полной взаимозаменяемостью деталей в узле выполняется условие (21), [1]

$$
\left.\begin{array}{l}
\sum_{l=1}^{i-1}(-1)^{n_{k, t}} r d i_{k, l}+(-1)^{n_{k, i}} r d i_{k, i}+\sum_{m=i+1}^{s-1}(-1)^{n_{k, m}} r d i_{k, m}=r d i_{k, s}, \\
\sum_{l=1}^{i-1}(-1)^{n_{k, t}} r d a_{k, l}+(-1)^{n_{k, t}} r d a_{k, i}+\sum_{m=i+1}^{s-1}(-1)^{n_{k, m}} r d a_{k, m}=r d a_{k, s}, \\
\sum_{l=1}^{i-1}(-1)^{n_{k, t}} r d n_{k, l}+(-1)^{n_{k, i}} r d n_{k, i}+\sum_{m=i+1}^{s-1}(-1)^{n_{k, m}} r d n_{k, m}=r d n_{k, s},
\end{array}\right\}
$$

где: $r d i, r d a, r d n$ - значения минимального, максимального и номинального размеров; индекс $k$ указывает на номер формируемой КРЦ; индексы $l, i, m-$ указывают на номера деталей, поверхности которых участвуют в формировании $k$-й КРЦ до $i$-й детали, $i$-ю деталь и после $i$-й детали соответственно; индекс $s$ указывает на номер замыкающего звена в $k$-й КРЦ; $(-1)^{n_{k, l}},(-1)^{n_{k, i}},(-1)^{n_{k, m}}-$ - знаки, указывающие на то, являются ли звенья увеличивающими $\left(1, n_{k, l}=0\right)$ или уменьшающими $\left(-1, n_{k, l}=1\right)$.

Рассмотрим варианты формирования КРЦ в зависимости от последовательности присоединения і-й детали к узлу:

1. Если первый размер $\left(r d_{k, i}, i=1\right)$ в $\kappa$-й КРЦ $\left(r d_{k, j}\right)$ формируется с использованием поверхностей $i$-й базовой детали, тогда система уравнений баланса размерной цепи (21) преобразуется к виду (22)

$$
\left.\begin{array}{l}
(-1)^{n_{k, i}} r d i_{k, i}+\sum_{m=i+1}^{s-1}(-1)^{n_{k, m}} r d i_{k, m}=r d i_{k, s}, \\
(-1)^{n_{k, i}} r d a_{k, i}+\sum_{m=i+1}^{s-1}(-1)^{n_{k, m}} r d a_{k, m}=r d a_{k, s}, \\
(-1)^{n_{k, i}} r d n_{k, i}+\sum_{m=i+1}^{s-1}(-1)^{n_{k, m}} r d n_{k, m}=r d n_{k, s},
\end{array}\right\} .
$$

2. Если последний размер $\left(r d_{k, i}, i=i-1\right)$ в $\kappa$-й КРЦ формируется с использованием поверхностей $i$-й базовой детали, тогда система уравнений баланса размерной цепи (21) преобразуется к виду (23)

$$
\left.\begin{array}{l}
\sum_{l=1}^{i-1}(-1)^{n_{k, l}} r d i_{k, l}+(-1)^{n_{k, i}} r d i_{k, i}=r d i_{k, s}, \\
\sum_{l=1}^{i-1}(-1)^{n_{k, l}} r d a_{k, l}+(-1)^{n_{k, i}} r d a_{k, i}=r d a_{k, s}, \\
\sum_{l=1}^{i-1}(-1)^{n_{k, l}} r d n_{k, l}+(-1)^{n_{k, i}} r d n_{k, i}=r d n_{k, s},
\end{array}\right\} .
$$

3. Если размер замыкающего звена $\left(r d_{k, i} i=s\right)$ в $\kappa$-й КРЦ формируется с использованием поверхностей $i$-й базовой детали, тогда система уравнений баланса размерной цепи (21) преобразуется к виду (24)

$$
\left.\begin{array}{l}
\sum_{l=1}^{s-1}(-1)^{n_{k, l}} r d i_{k, l}=r d i_{k, i}, \\
\sum_{l=1}^{s-1}(-1)^{n_{k, l}} r d a_{k, l}=r d a_{k, i}, \\
\sum_{l=1}^{s-1}(-1)^{n_{k, l}} r d n_{k, l}=r d n_{k, i},
\end{array}\right\} .
$$

Концепция механической обработки резанием деталей узла состоит в том, чтобы РКЦ рассчитывать с учетом синхронизации процессов сборки изделия и обработки его деталей, рис. 1, ф-ла (8).

При обработке $i$-й базовой детали, в соответствии с этой концепцией, все детали $(l=\overline{1, i-1})$, собранные в виртуальном узле до ее установки в изделие (рис. 1), обработаны и имеют конкретные значения размеров. Тогда в системах уравнений $(21,23,24)$ вместо максимального $\left(r d a_{k, l}\right)$ и минимального $\left(r d i_{k, l}\right)$ размеров звеньев КРЦ должны быть заданы номинальные размеры поверхностей обработанных деталей, участвующих в формировании звеньев $k$-й КРЦ:

1. Тогда (21) преобразуется к виду (25)

$$
\left.\begin{array}{l}
\sum_{l=1}^{i-1}(-1)^{n_{k, t}} r d n_{k, l}+(-1)^{n_{k, i}} r d i_{k, i}+\sum_{m=i+1}^{s-1}(-1)^{n_{k, m}} r d i_{k, m}=r d i_{k, s}, \\
\sum_{l=1}^{i-1}(-1)^{n_{k, t}} r d n_{k, l}+(-1)^{n_{k, i}} r d d_{k, i}+\sum_{m=i+1}^{s-1}(-1)^{n_{k, m}} r d a_{k, m}=r d a_{k, s}, \\
\sum_{l=1}^{i-1}(-1)^{n_{k, t}} r d n_{k, l}+(-1)^{n_{k, i}} r d n_{k, i}+\sum_{m=i+1}^{s-1}(-1)^{n_{k, m}} r d n_{k, m}=r d n_{k, s},
\end{array}\right\} .
$$

2. Тогда (23) преобразуется к виду (26)

$$
\left.\begin{array}{l}
\sum_{l=1}^{i-1}(-1)^{n_{k, l}} r d n_{k, l}+(-1)^{n_{k, l}} r d i_{k, i}=r d i_{k, s}, \\
\sum_{l=1}^{i-1}(-1)^{n_{k, l}} r d n_{k, l}+(-1)^{n_{k, i}} r d a_{k, i}=r d a_{k, s}, \\
\sum_{l=1}^{i-1}(-1)^{n_{k, l}} r d n_{k, l}+(-1)^{n_{k, i}} r d n_{k, i}=r d n_{k, s},
\end{array}\right\} .
$$


3. Тогда (24) преобразуется к виду (27)

$$
\left.\begin{array}{l}
\sum_{l=1}^{s-1}(-1)^{n_{k, l}} r d n_{k, l}=r d i_{k, i}, \\
\sum_{l=1}^{s-1}(-1)^{n_{k, l}} r d n_{k, l}=r d a_{k, i}, \\
\sum_{l=1}^{s-1}(-1)^{n_{k, l}} r d n_{k, l}=r d n_{k, i},
\end{array}\right\} .
$$

или

$$
\sum_{l=1}^{s-1}(-1)^{n_{k, l}} r d n_{k, l}=r d n_{k, i}
$$

Тогда при изготовлении $i$-й базовой детали, ее размеры, входящие в $k$-ю КРЦ, определяются из следующих выражений:

1. Если первый размер $\left(r d_{k, i}, i=1\right)$ в $\kappa$-й КРЦ формируется с использованием поверхностей $i$-й базовой детали. Тогда из (22) получим выражения для расчёта размеров обработки (29)

$$
\left.\begin{array}{c}
(-1)^{n_{k, i}} r d i_{k, i}=r d i_{k, s}-\sum_{m=i+1}^{s-1}(-1)^{n_{k, m}} r d i_{k, m}, \\
(-1)^{n_{k, i}} r d a_{k, i}=r d a_{k, s}-\sum_{m=i+1}^{s-1}(-1)^{n_{k, m}} r d a_{k, m}, \\
(-1)^{n_{k, i}} r d n_{k, i}=r d n_{k, s}-\sum_{m=i+1}^{s-1}(-1)^{n_{k, m}} r d n_{k, m} \cdot
\end{array}\right\} .
$$

2. Если промежуточный размер $\left(r d_{k, i}, i>1 \wedge i<\right.$ $<n-1)$ в $\kappa$-й КРЦ формируется с использованием поверхностей $i$-й базовой детали. Тогда из (21) получим выражения для расчёта размеров обработки (30)

$$
\left.\begin{array}{l}
(-1)^{n_{k, t}} r d i_{k, i}=r d i_{k, s}-\sum_{l=1}^{i-1}(-1)^{n_{k, t}} r d i_{k, l}-\sum_{m=i+1}^{s-1}(-1)^{n_{k, m}} r d i_{k, m}, \\
(-1)^{n_{k, i}} r d a_{k, i}=r d a_{k, s}-\sum_{l=1}^{i-1}(-1)^{n_{k, t}} r d a_{k, l}-\sum_{m=i+1}^{s-1}(-1)^{n_{k, m}} r d a_{k, m}, \\
(-1)^{n_{k, i}} r d n_{k, i}=r d n_{k, s}-\sum_{l=1}^{i-1}(-1)^{n_{k, t}} r d n_{k, l}-\sum_{m=i+1}^{s-1}(-1)^{n_{k, m}} r d n_{k, m}
\end{array}\right\} .
$$

3. Если последний размер в к-й КРЦ формируется с использованием поверхностей i-й базовой детали. Тогда из (26) получим выражения для расчёта размеров обработки (31)

$$
\left.\begin{array}{l}
(-1)^{n_{k, i}} r d i_{k, i}=r d i_{k, s}-\sum_{l=1}^{i-1}(-1)^{n_{k, l}} r d n_{k, l}, \\
(-1)^{n_{k, i}} r d a_{k, i}=r d a_{k, s}-\sum_{l=1}^{i-1}(-1)^{n_{k, l}} r d n_{k, l}, \\
(-1)^{n_{k, i}} r d n_{k, i}=r d n_{k, s}-\sum_{l=1}^{i-1}(-1)^{n_{k, l}} r d n_{k, l} \cdot
\end{array}\right\} .
$$

4. Если размеры $i$-й детали $\left(r d_{k, i}, i=n-1\right)$ в $\kappa$-й КРЦ являются замыкающим звеном, тогда размеры обработки ее поверхностей можно получить из (28).

В соответствии со схемой (рис. 1), на третьем этапе сборки ВИ без селективного отбора определим размерные параметры процесса формообразования поверхностей $i$-й базовой детали в виде технологических размерных цепей (ТРЦ).

Нас будут интересовать только та $t$-я ТРЦ $\left(t r c_{t}\right)$ на этапе окончательного формирования размеров детали, входящие в $\kappa$-ю КРЦ $\left(k r c_{k}\right)(32)$.

$$
\left(r d i_{k, i} \in k r c_{k}\right) \wedge r d i_{k, i} \in\left(r d i_{k, i} \in t r c_{t}\right) .
$$

Известно, что в ТРЦ в качестве замыкающего звена используется снимаемый припуск материала снимаемого на переходе $(\Delta)$. Тогда система уравнений, определяющих его номинальный $(\Delta \mathrm{n})$, минимальный $(\Delta \mathrm{i})$ и максимальный $(\Delta \mathrm{a})$ размеры, имеет вид (33), [1]

$$
\left.\begin{array}{l}
\sum_{l=1}^{u_{s}}(-1)^{n_{s, l}} d n_{s, l}-\sum_{m=1}^{p_{t, m}}(-1)^{n_{t, m}} r z a_{t, m}=\Delta \mathrm{i}, \\
\sum_{l=1}^{u_{s}}(-1)^{n_{s, l}} d n_{s, l}-\sum_{m=1}^{p_{t, m}}(-1)^{n_{t, m}} r z i_{t, m}=\Delta \mathrm{a}, \\
\sum_{l=1}^{u_{s}}(-1)^{n_{s, l}} d n_{s, l}-\sum_{m=1}^{p_{t, m}}(-1)^{n_{t, m}} r z n_{t, m}=\Delta \mathrm{n},
\end{array}\right\} .
$$

где: $d n_{s, l}-l$-й номинальный размер $s$-й детали, полученный на предыдущих этапах обработки и участвующий в формировании $t$-й ТРЦ, замыкающим звеном которой является номинальный размера $\mathrm{r} d n_{k, s}$ участвующий в формировании $\kappa$-й КРЦ; $r z i_{t, m}$, $r z a_{t, m}, r z n_{t, m}$ - минимальное, максимальное и номинальное значения размера заготовки $(m=\overline{1, p})$ для $(k, s)$-го размера поверхности детали; $u_{s}-$ количество размеров $s$-й детали, сформированных до получения размера $r d n_{k, s} ; p_{s}-$ количество промежуточных размеров заготпвок, из которых формируется размер $r d n_{k, s} ;(-1)^{n_{s, l}},(-1)^{n_{i, m}}$ - формулы, определяющие знаки звеньев, составляющих $t$-ю ТРЦ: собственных размеров детали и размеров заготовки на различных этапах обработки.

С учетом того, что нас интересует только переход, на котором формируется размер детали, заданный конструктором $\left(r d i_{k, s} r d a_{k, s} r d n_{k, s}\right)$, размеры припуска на обработку $(\Delta \mathrm{i}, \Delta \mathrm{a}, \Delta \mathrm{n})$ можно представить в виде разности размеров детали и размеров заготовки до обработки на последнем переходе (34)

$$
\begin{aligned}
& \Delta \mathrm{i}=\sum_{l=1}^{u_{s}}(-1)^{n_{s, l}} d n_{s, l}-(-1)^{n_{t, t, t, j}} r z a_{t, p_{t, j}}, \\
& \left.\Delta \mathrm{a}=\sum_{l=1}^{u_{s}}(-1)^{n_{s, l}} d n_{s, l}-(-1)^{n_{t, p, j}} r z i_{t, p_{t, j}},\right\} . \\
& \Delta \mathrm{n}=\sum_{l=1}^{u_{s}}(-1)^{n_{s, l}} d n_{s, l}-(-1)^{n_{t, p t, j}} r z n_{t, p_{t, j}},
\end{aligned}
$$


Тогда в $t$-ю ТРЦ, по аналогии с $\kappa$-й КРЦ, в качестве звеньев входят: замыкающее звено - формируемый размер детали $\left(r d_{k, s}\right)$, составляющие звенья конструкторские размеры детали $\left(d n_{s, l}\right)$, промежуточные технологические размеры заготовки $\left(r z n_{t, p}\right)$. А ф-ла (33) учетом (34)преобразуется к виду (35)

$$
\left.\begin{array}{l}
r d i_{k, s}=\sum_{l=1}^{u_{s}}(-1)^{n_{s, l}} d n_{s, l}-(-1)^{n_{t, j}} r z_{t, p_{t, j}}, \\
r d a_{k, s}=\sum_{l=1}^{u_{s}}(-1)^{n_{s, l}} d n_{s, l}-(-1)^{n_{t, j}} r z_{t, p_{t, j}} \\
r d n_{k, s}=\sum_{l=1}^{u_{s}}(-1)^{n_{s, l}} d n_{s, l}-(-1)^{n_{t, j}} r z_{t, p_{t, j}},
\end{array}\right\} .
$$

Следовательно, при иерархической обработке деталей изделия, на последнем переходе механической обработки ее заготовки необходимо вводить коррекцию допуска на соответствующий размер заготовки (36)

Исходя из этого, задачей технолога будет формирование технологических процессов получения размеров деталей таким образом, чтобы они учитывали последовательность сборки изделия и были с нею синхронизированы, а также обеспечивали заданные погрешности КРЦ.

\section{Практическая реализация}

Рассмотрим методику автоматизированного механосборочного производства высокоточных машиностроительных изделий на основе интеграции производственных процессов на примере моделирования сборки и обработки деталей узла «Механизм быстрого подвода каретки шлифовальной бабки» модели ХШ5-20М.920-1, рис. 2.

Узел предназначен для поперечного ускоренного перемещения шлифовальной бабки относительно подкладной плиты.

Он состоит: из узла «Передача винт-гайка качения» (1), винт которого закреплен на подкладной плите с помощью подшипников скольжения (12) и (25); состоит из червяка (30), установленного в корпусе (10) на подшипниках (64), который передает крутящий момент от вала (9), соединенного с электродвигателем на червячное колесо узла (1); для предотвращения изгиба винта узла (1) от нагрузок в червячной передаче применена разгруженная схема крепления червячного колеса с использование шариковых радиальных подшипников (65) и упорного подшипника (63); регулирование натяга в передаче винт-гайка качения осуществляется путем поворота в противоположные стороны относительно оси винта (1) двух зубчатых муфт с их последующей фиксацией; подача смазки в передачу червяк - червячное колесо осуществ- ляется через штуцер (18) по трубопроводу (17), а в передачу винт-гайка качения - по трубопроводу (16); крепление корпуса механизма быстрого подвода к шпиндельной бабке осуществляется болтами (42), а его базирование - штифтами (57); для обеспечения точного перемещения шпиндельной бабки в механизме быстрого подвода использован следящий привод, состоящий из рейки, закрепленной на шпиндельной бабке и направляющего механизма, состоящего из кронштейна (13), на котором крепятся два ролика (32), один из которых расположен на рычаге (19) и поджимается к рейке упором (20); регулировка усилия поджима осуществляется пружиной (21) и пробкой (70).

Приведенная выше модель механосборочного процесс (формулы 1-35) реализована в виде алгоритма и программы расчетов в среде Mathcad 15:

На первом этапе по данным сборочного чертежа изделия и параметрам деталей, входящих в него (рис. 2) формируется матрица смежности деталей, входящих в изделие (рис. 3)

На втором этапе, по (7) рассчитывается комплексный коэффициент эффективности каждой базовой детали (в алгоритме он назван коэффициентом сборки - $K s b)$, рис. 4. Пример такого расчета представлен на рис. 5, а последовательность сборки изделия - в таблице 1.

На третьем этапе формируется последовательность сборки изделия (рис. 6).

Алгоритм организации последовательности сборки изделия (Рог) на основе расчета коэффициента сборки $K s b$ состоит из следующих этапов:

1. Определяем 0-ю базовую детали исходя из того, что значение $K s b$ для нее должно быть максимальным (параметры $P_{0 p z_{i, 0}}$ - номер детали;

Poг $_{i, 1}$ - деталь, к которой она присоединяется:

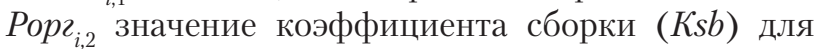
данной детали);

2. Получаем 0 -й этап сборки.

Для поиска детали, подходящей для последующего этапа сборки выполняем следующие операции:

3. Для всех, уже установленных в узел, деталей находим детали, которые еще можно к ним присоединить.

4. Для каждой из них определяем коэффициент сборки.

5. Находим базируемые детали с максимальным значением коэффициента сборки. Найденные детали устанавливаются поэтапно в порядке их обнаружения.

6. После добавления в сборку очередной детали проверяют условие крепления деталей между собой. Если оно выполняется, добавляю крепежную деталь с $\max (K s b)$.

7. Этапов установки в узел деталей столько, сколько деталей в узле (включая крепежные). 


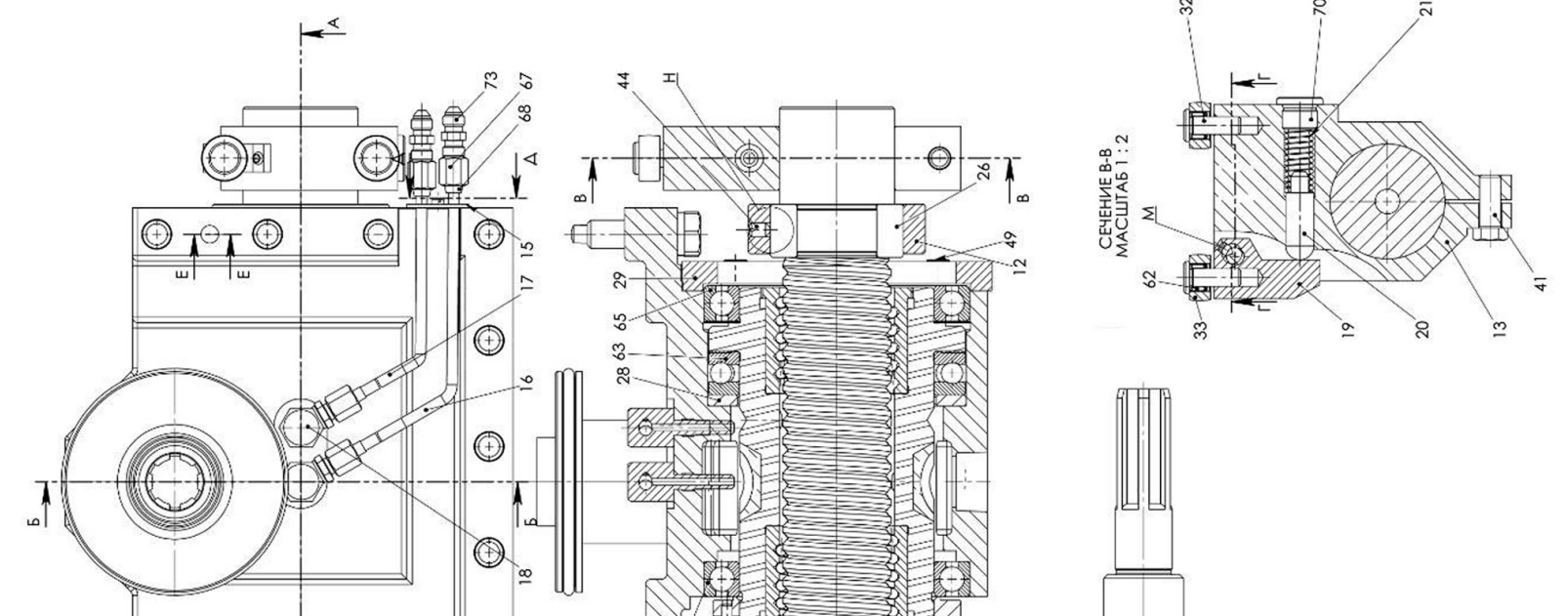

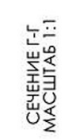

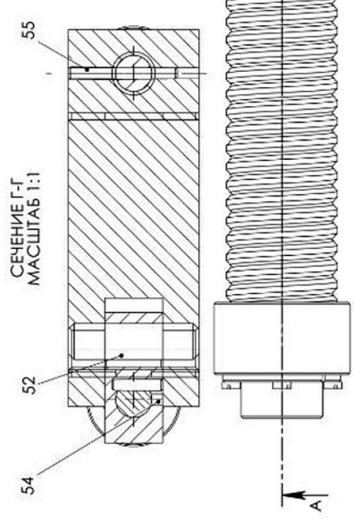

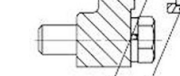
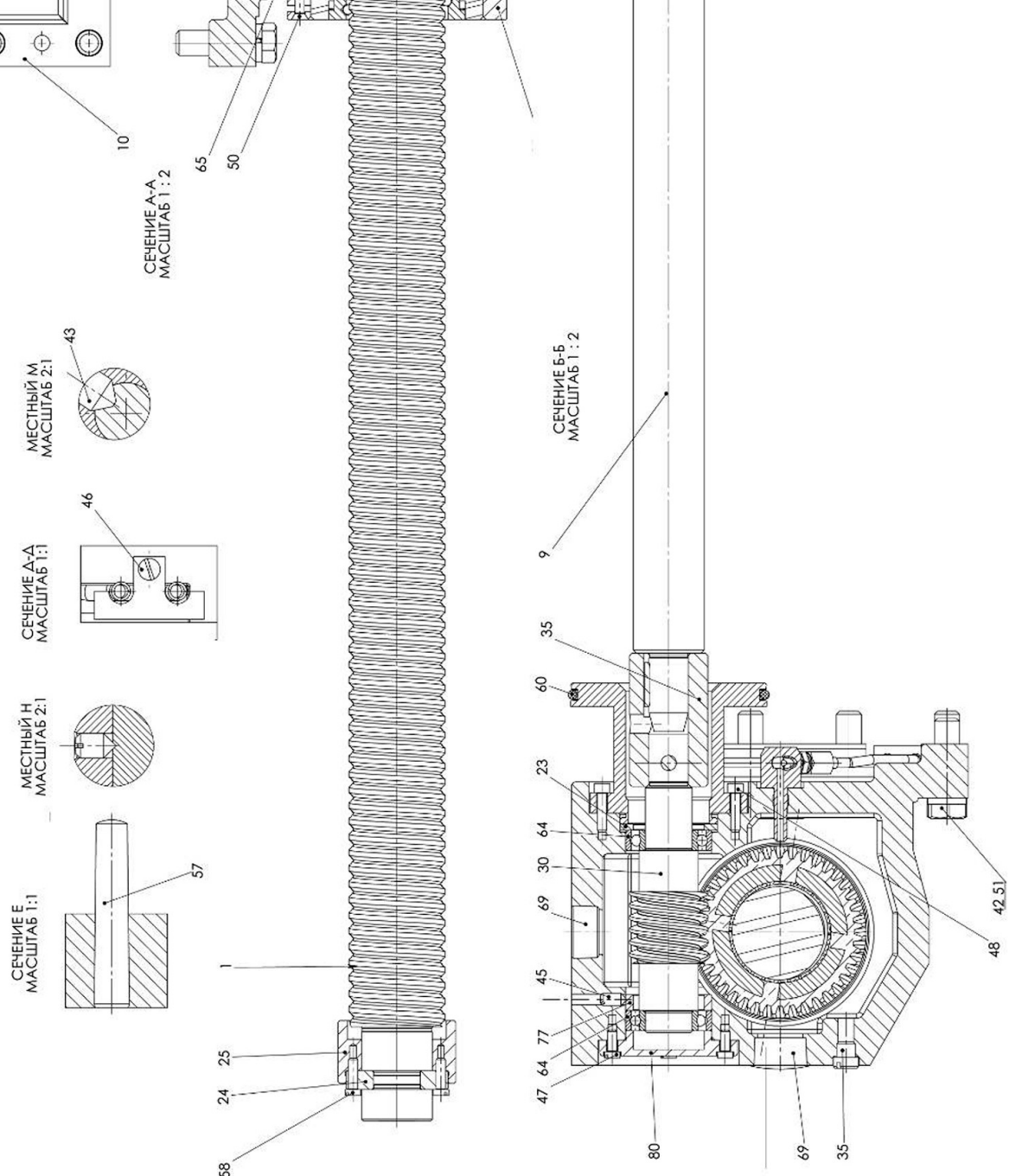


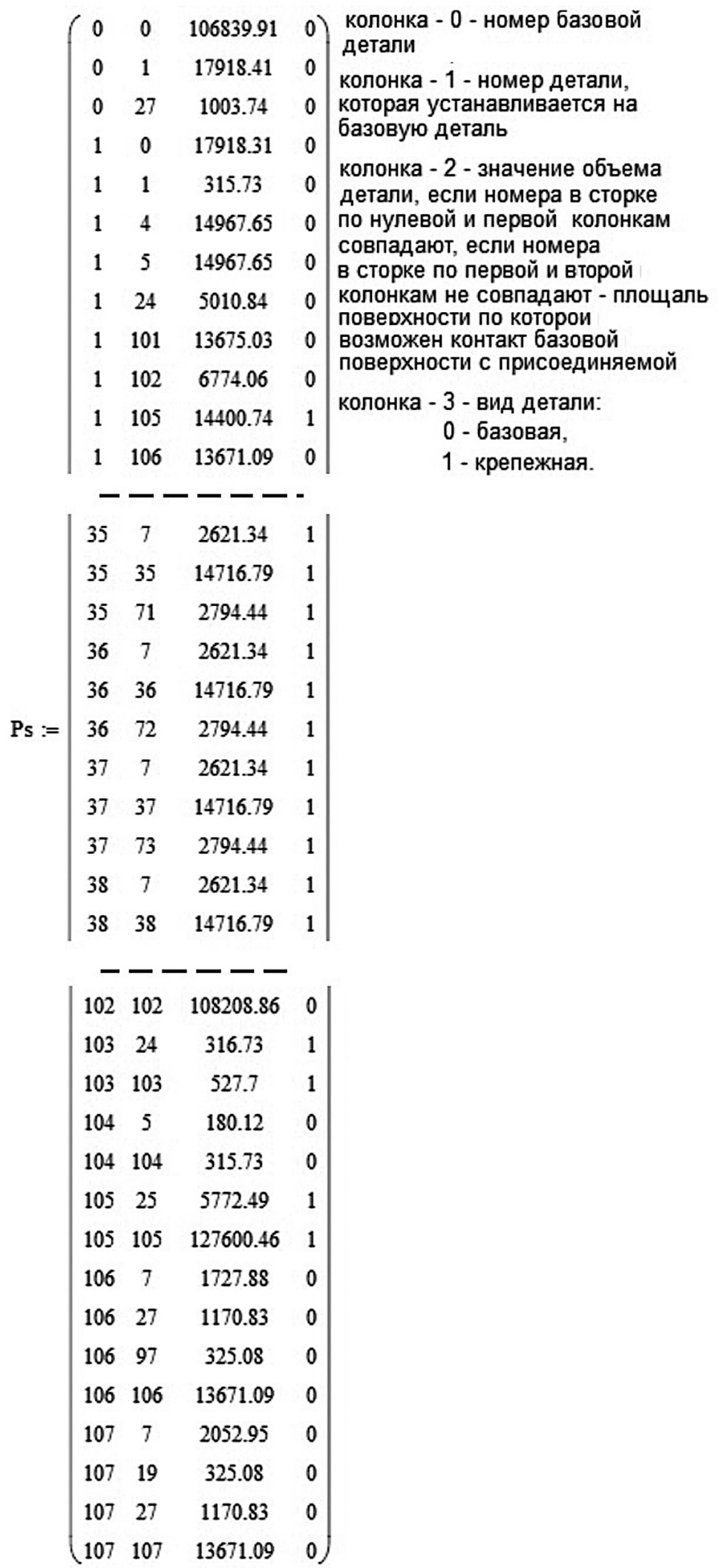

$\begin{array}{llll}103 & 24 & 316.73 & 1\end{array}$

$\begin{array}{llll}103 & 103 & 527.7 & 1\end{array}$

$\begin{array}{llll}104 & 5 & 180.12 & 0\end{array}$

$\begin{array}{llll}104 & 104 & 315.73 & 0\end{array}$

$\begin{array}{llll}105 & 25 & 5772.49 & 1\end{array}$

$\begin{array}{llll}105 & 105 & 127600.46 & 1\end{array}$

$106-727.88$

$\begin{array}{llll}106 & 27 & 1170.83\end{array}$

$\begin{array}{llll}106 & 97 & 325.08 & 0\end{array}$

$\begin{array}{llll}106 & 106 & 13671.09 & 0\end{array}$

$\begin{array}{llll}107 & 7 & 2052.95 & 0\end{array}$

$\begin{array}{llll}07 & 19 & 325.08 & 0\end{array}$

$\begin{array}{llll}107 & 107 & 13671.09 & 0\end{array}$

Рис. 3. Фрагмент матрицы смежности поверхностей деталей узла «Механизм быстрого подвода каретки шлифовальной бабки» 


$$
\begin{aligned}
& \text { Ksb }:=\mid i \leftarrow 0 \\
& \mathrm{v}_{\mathrm{i}} \leftarrow 0 \\
& \mathrm{~S}_{\mathrm{i}} \leftarrow 0 \\
& n \max \leftarrow 0 \\
& n_{i} \leftarrow 0 \\
& S \max \leftarrow 0 \\
& V_{\max } \leftarrow 0 \\
& \text { kstr } \leftarrow \text { rows }(\mathrm{Ps}) \\
& \text { for } k \in 0,1 . . k s t r-1 \\
& \text { if } \mathrm{Ps}_{\mathrm{k}, 0}>\mathrm{i} \\
& i \leftarrow i+1 \\
& \mathrm{v}_{\mathrm{i}} \leftarrow 0 \\
& \mathrm{~s}_{\mathrm{i}} \leftarrow 0 \\
& n_{\mathrm{i}} \leftarrow 0 \\
& \mathrm{~V}_{\mathrm{i}} \leftarrow \mathrm{Ps}_{\mathrm{k}, 2} \text { if } \mathrm{Ps}_{\mathrm{k}, 0}=\mathrm{Ps}_{\mathrm{k}, 1} \\
& \text { if } \mathrm{Ps}_{\mathrm{k}, 3}=0 \wedge \mathrm{Ps}_{\mathrm{k}, 0} \neq \mathrm{Ps}_{\mathrm{k}, 1} \\
& \mid \begin{array}{l}
\mathrm{S}_{\mathrm{i}} \leftarrow \mathrm{S}_{\mathrm{i}}+\mathrm{Ps}_{\mathrm{k}, 2} \\
\mathrm{n}_{\mathrm{i}} \leftarrow \mathrm{n}_{\mathrm{i}}+1
\end{array}
\end{aligned}
$$

\begin{tabular}{|c|c|c|c|c|}
\hline & 0 & 1 & 2 & 3 \\
\hline 10 & $2.811 \cdot 10^{-4}$ & $1.521 \cdot 10^{-3}$ & 0.031 & 0.033 \\
\hline 11 & $7.595 \cdot 10^{-4}$ & 0.012 & 0.063 & 0.075 \\
\hline 12 & $6.012 \cdot 10^{-4}$ & 0.012 & 0.063 & 0.075 \\
\hline 13 & $3.22 \cdot 10^{-3}$ & $4.95 \cdot 10^{-3}$ & 0.063 & 0.071 \\
\hline 14 & $3.22 \cdot 10^{-3}$ & $4.95 \cdot 10^{-3}$ & 0.063 & 0.071 \\
\hline 15 & $6.011 \cdot 10^{-3}$ & 0.014 & 0.125 & 0.145 \\
\hline 16 & $2.283 \cdot 10^{-3}$ & $9.875 \cdot 10^{-3}$ & 0.094 & 0.106 \\
\hline 17 & $7.242 \cdot 10^{-4}$ & 0.018 & 0.063 & 0.081 \\
\hline 18 & 0.034 & 0.065 & 0.125 & 0.224 \\
\hline 19 & $1.612 \cdot 10^{-3}$ & 0.013 & 0.063 & 0.077 \\
\hline 20 & $8.286 \cdot 10^{-3}$ & 0.01 & 0.063 & 0.081 \\
\hline 21 & 0.023 & 0.024 & 0.063 & 0.109 \\
\hline 22 & 0.024 & 0.044 & 0.063 & 0.13 \\
\hline 23 & 0.027 & 0.026 & 0.031 & 0.084 \\
\hline 24 & 0.026 & 0.04 & 0.063 & 0.128 \\
\hline 25 & 0.012 & 0.034 & 0.031 & 0.077 \\
\hline 26 & 0.04 & 0.025 & 0.031 & 0.096 \\
\hline$\cdots$ & $\cdots$ & $\cdots$ & $\cdots$ & $\cdots$ \\
\hline 90 & $5.968 \cdot 10^{-5}$ & 0.177 & 0.031 & 0.208 \\
\hline 91 & $5.968 \cdot 10^{-5}$ & 0.177 & 0.031 & 0.208 \\
\hline 92 & $1.573 \cdot 10^{-3}$ & $4.445 \cdot 10^{-3}$ & 0.063 & 0.069 \\
\hline 93 & $3.676 \cdot 10^{-3}$ & $7.775 \cdot 10^{-3}$ & 0.031 & 0.043 \\
\hline 94 & $3.7 \cdot 10^{-3}$ & $6.873 \cdot 10^{-3}$ & 0.031 & 0.042 \\
\hline 95 & $1.278 \cdot 10^{-3}$ & $2.005 \cdot 10^{-3}$ & 0.063 & 0.066 \\
\hline 96 & $1.278 \cdot 10^{-3}$ & $2.005 \cdot 10^{-3}$ & 0.063 & 0.066 \\
\hline 97 & $2.226 \cdot 10^{-3}$ & $9.313 \cdot 10^{-3}$ & 0.063 & 0.074 \\
\hline 98 & 0.015 & 0.022 & 0.031 & 0.069 \\
\hline 99 & $7.23 \cdot 10^{-4}$ & $\mathbf{0}$ & $\mathbf{0}$ & $7.23 \cdot 10^{-4}$ \\
\hline 100 & $7.23 \cdot 10^{-4}$ & $\mathbf{0}$ & $\mathbf{0}$ & $7.23 \cdot 10^{-4}$ \\
\hline 101 & 0.031 & 0.073 & 0.094 & 0.197 \\
\hline 102 & 0.031 & 0.059 & 0.063 & 0.152 \\
\hline 103 & $1.511 \cdot 10^{-4}$ & $\mathbf{0}$ & $\mathbf{0}$ & $1.511 \cdot 10^{-4}$ \\
\hline 104 & $9.041 \cdot 10^{-5}$ & $7.682 \cdot 10^{-4}$ & 0.031 & 0.032 \\
\hline 105 & 0.037 & 0 & $\mathbf{0}$ & 0.037 \\
\hline 106 & $3.915 \cdot 10^{-3}$ & 0.014 & 0.094 & 0.111 \\
\hline 107 & $3.915 \cdot 10^{-3}$ & 0.015 & 0.094 & 0.113 \\
\hline
\end{tabular}

Рис. 4. Алгоритм расчета коэффициента сборки деталей в узле $(K s b)$ Vmax - наибольшая объем (масса) детали в изделии;

Smax - наибольшее значение суммарной площади возможного контакта детали с другими деталями в узле;

$N \max$ - наибольшее количество контактов детали с другими деталями в узле $\mid \begin{aligned} & \text { for } i \in 0,1 \ldots s_{k s t r}-1,0 \\ & \mid \begin{array}{l}V \max \leftarrow V_{i} \text { if } V_{i}>V \max \\ S \max \leftarrow S_{i} \text { if } S_{i}>S \max \\ n \max \leftarrow n_{i} \text { if } n_{i}>n \max \end{array}\end{aligned}$

$$
\mid \begin{aligned}
& \mathrm{Kc}_{\mathrm{m}, 0} \leftarrow \frac{\mathrm{V}_{\mathrm{m}}}{\mathrm{V}_{\max }} \\
& \mathrm{K} \mathrm{c}_{\mathrm{m}, 1} \leftarrow \frac{\mathrm{S}_{\mathrm{m}}}{\mathrm{Smax}} \\
& \mathrm{K} \mathrm{c}_{\mathrm{m}, 2} \leftarrow \frac{\mathrm{n}_{\mathrm{m}}}{\mathrm{nmax}} \\
& \mathrm{K} \mathrm{c}_{\mathrm{m}, 3} \leftarrow \mathrm{Kc} \mathrm{m}_{\mathrm{m}, 0}+\mathrm{K} \mathrm{c}_{\mathrm{m}, 1}+\mathrm{K} \mathrm{c}_{\mathrm{m}, 2}
\end{aligned}
$$

$\mathrm{Ksb} \leftarrow \mathrm{Kc}$ for $m \in 0,1 .$. Ps $_{\text {kstr }-1,0}$
Матрица Ksb, состоящая из:

0-я колонка коэффиценты

$$
\frac{\mathrm{V}_{\mathrm{m}}}{\mathrm{V}_{\max }}
$$

для всех деталей

входящих в сборку;

1-я колонка коэффиценты

$$
\frac{\mathrm{S}_{\mathrm{m}}}{\mathrm{Smax}}
$$

для всех деталей,

входящих в сборку;

2-я колонка коэффиценты

$$
\frac{\mathrm{n}_{\mathrm{m}}}{\mathrm{nmax}}
$$

для всех деталей,

входящих в сборку.

3-я колонка коэффиценты

$\frac{\mathrm{V}_{\mathrm{m}}}{\mathrm{V}_{\max }}+\frac{\mathrm{S}_{\mathrm{m}}}{\mathrm{S} \max }+\frac{\mathrm{n}_{\mathrm{m}}}{\mathrm{n} \max }$

для всех деталей,

входящих в сборку.

Рис. 5. Пример расчета коэффициента сборки (Ksb) для деталей, входящих в узел «Механизм быстрого подвода каретки шлифовальной бабки» 


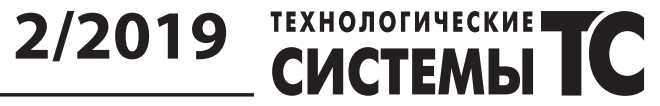

Таблица 1.

Фрагмент схемы сборки узла «Механизм быстрого подвода каретки шлифовальной бабки»

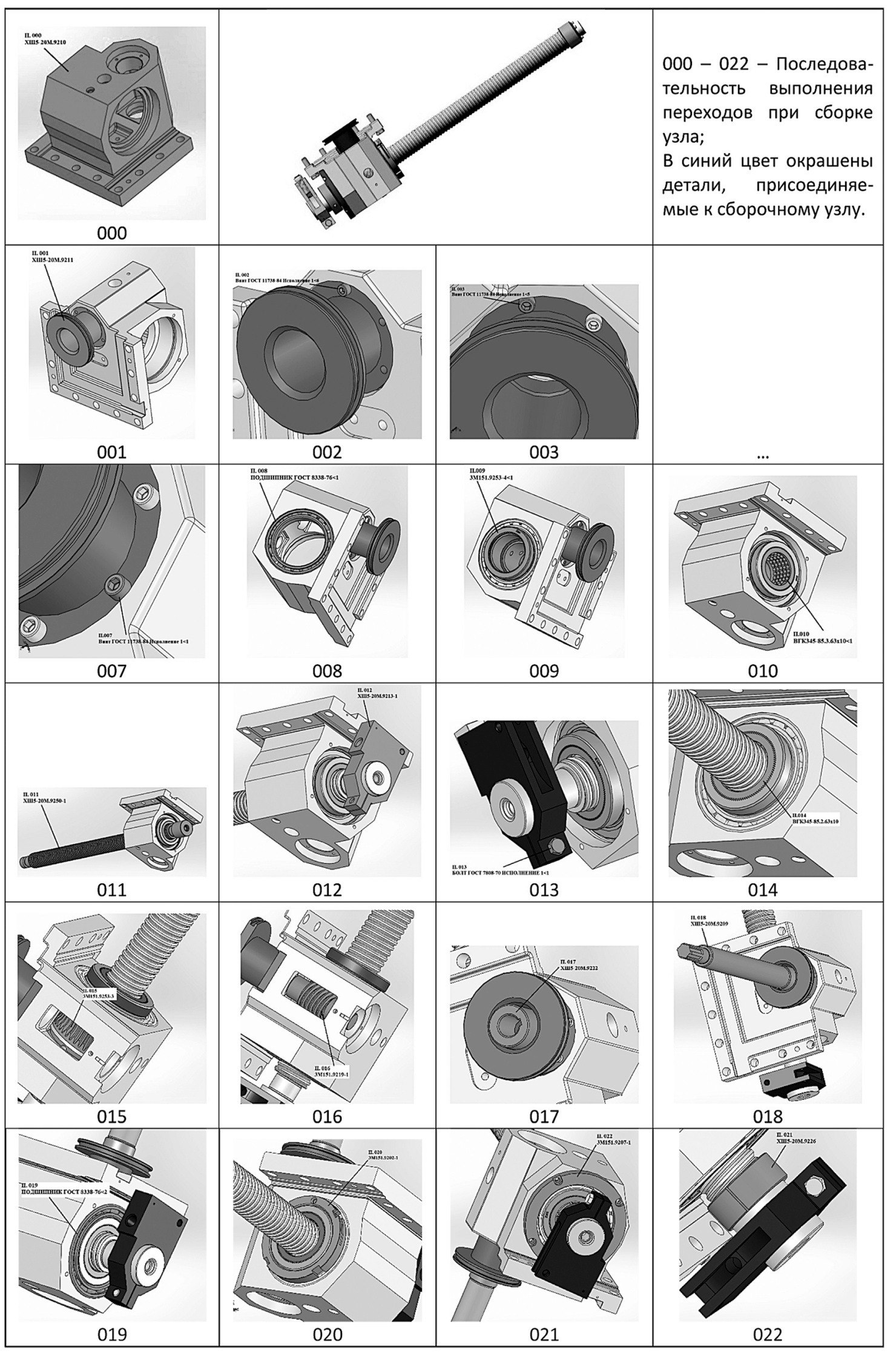



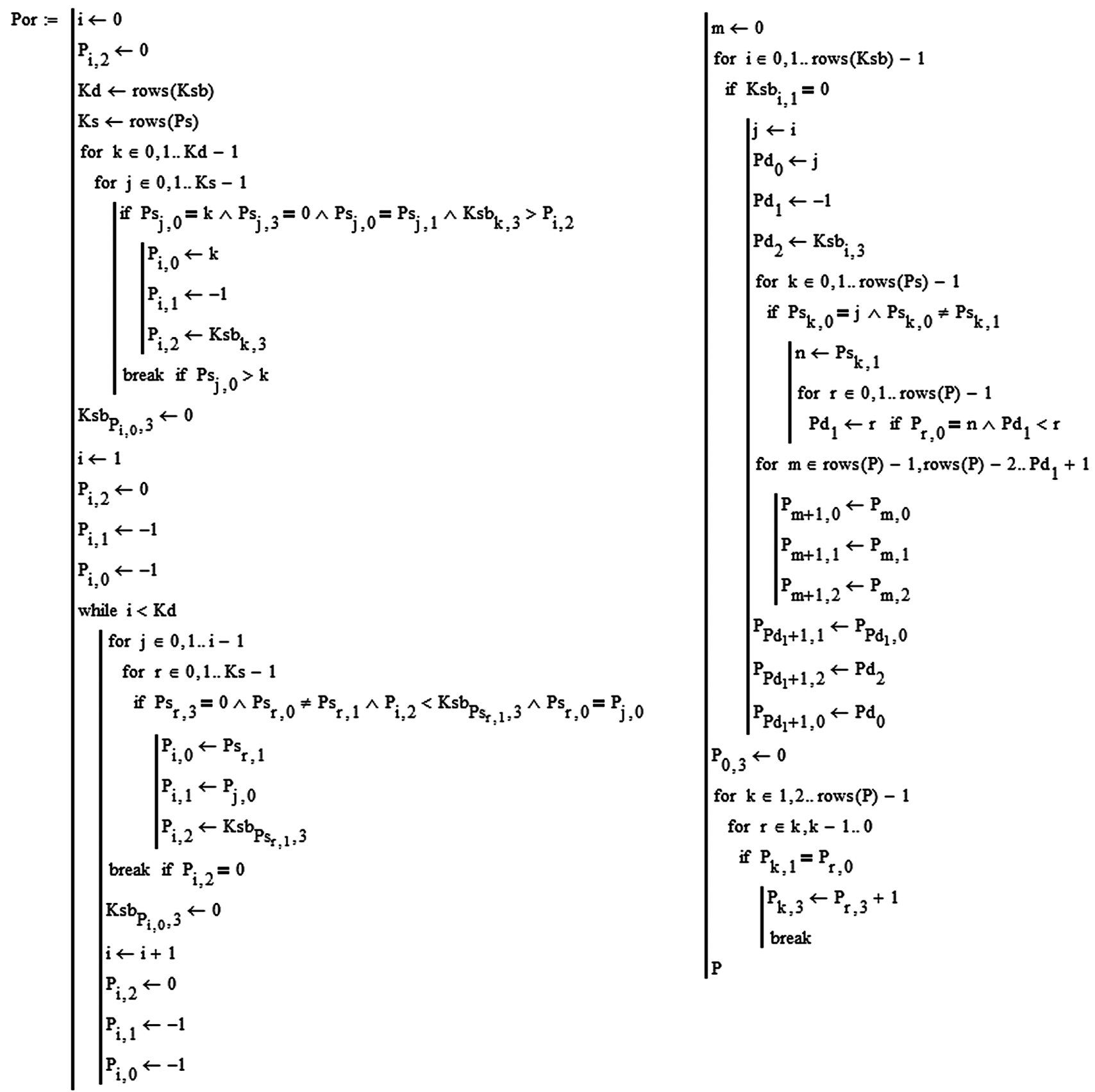

Рис. 6. Алгоритм организации последовательности сборки изделия

Фрагмент таблицы формирования последовательности сборки узла «Механизм быстрого подвода каретки шлифовальной бабки», сформированной по алгоритму (рис. 6), представлен на рис. 7.

Алгоритм поиска последней базовой детали в дереве построения технологического процесса сборки изделия, с которой соединяется крепежная деталь, представлен на рис. 8. С его помощью была сформирована таблица возможной последовательности параллельно-последовательной сборки узла «Механизм быстрого подвода каретки шлифовальной бабки» (рис. 9). 


\begin{tabular}{|c|c|c|c|c|}
\hline & 0 & 1 & 2 & 3 \\
\hline 0 & 7 & -1 & 2.756 & 0 \\
\hline 1 & 101 & 7 & 0.197 & 1 \\
\hline 2 & 1 & 101 & 0.59 & 2 \\
\hline 3 & 5 & 1 & 0.252 & 3 \\
\hline 4 & 2 & 5 & 1.947 & 4 \\
\hline 5 & 9 & 2 & 0.379 & 5 \\
\hline 6 & 33 & 9 & $4.214 \cdot 10^{-3}$ & 6 \\
\hline 7 & 4 & 1 & 0.252 & 3 \\
\hline 8 & 8 & 7 & 0.193 & 1 \\
\hline 9 & 62 & 8 & $3.099 \cdot 10^{-4}$ & 2 \\
\hline 10 & 61 & 8 & $3.099 \cdot 10^{-4}$ & 2 \\
\hline 11 & 60 & 8 & $3.099 \cdot 10^{-4}$ & 2 \\
\hline 12 & 59 & 8 & $3.099 \cdot 10^{-4}$ & 2 \\
\hline 13 & 58 & 8 & $3.099 \cdot 10^{-4}$ & 2 \\
\hline 14 & 57 & 8 & $3.099 \cdot 10^{-4}$ & 2 \\
\hline$\cdots$ & $\cdots$ & $\cdots$ & $\cdots$ & $\cdots$ \\
\hline 92 & 74 & 7 & 0.033 & 1 \\
\hline 93 & 38 & 74 & $4.214 \cdot 10^{-3}$ & 2 \\
\hline 94 & 75 & 7 & 0.033 & 1 \\
\hline 95 & 39 & 75 & $4.214 \cdot 10^{-3}$ & 2 \\
\hline 96 & 76 & 7 & 0.033 & 1 \\
\hline 97 & 40 & 76 & $4.214 \cdot 10^{-3}$ & 2 \\
\hline 98 & 77 & 7 & 0.033 & 1 \\
\hline 99 & 41 & 77 & $4.214 \cdot 10^{-3}$ & 2 \\
\hline 100 & 78 & 7 & 0.033 & 1 \\
\hline 101 & 42 & 78 & $4.214 \cdot 10^{-3}$ & 2 \\
\hline 102 & 79 & 7 & 0.033 & 1 \\
\hline 103 & 43 & 79 & $4.214 \cdot 10^{-3}$ & 2 \\
\hline 104 & 80 & 7 & 0.033 & 1 \\
\hline 105 & 44 & 80 & $4.214 \cdot 10^{-3}$ & 2 \\
\hline 106 & 104 & 5 & 0.032 & 4 \\
\hline 107 & 3 & 4 & 0.032 & 4 \\
\hline 108 & -1 & -1 & 0 & 1 \\
\hline
\end{tabular}

колонка - 0 - номер устанавливаемой детали колонка - 1 - номер детали, на которую устанавливается деталь

колонка - 2 - значение коэффициента сборки (Ksb) дпя данной детали номер строки сверху вниз - этапы сборки изделия (0-й, 1-й, 2-й и т.д. Кd-й, который соответсвует количеству деталей в собираемом узле)

колонка - 2 - уровень, на котором может

выполняться переход соединения детали с номером

из 0-й колонки с деталью из 1-й колонки

("-1" обозначает, что соединения нет).

Pис. 7. Фрагмент таблицы формирования последовательности сборки узла «Механизм быстрого подвода каретки шлифовальной бабки»

С учетом данных таблицы (рис. 7), получим таблицу структуры ТП параллельно-последовательной сборки узла (табл. 2).

В приведенном примере (таб. 1) представлены переходы ТП сборки изделия, которые могут выполняться независимо друг от друга в пределах одного уровня ТП сборки (таб. 2), а их последовательность выполнения регламентирована последовательностью уровней сборки. 
Структура ТП параллельно-последовательной сборки узла «Механизм быстрого подвода каретки шлифовальной бабки»

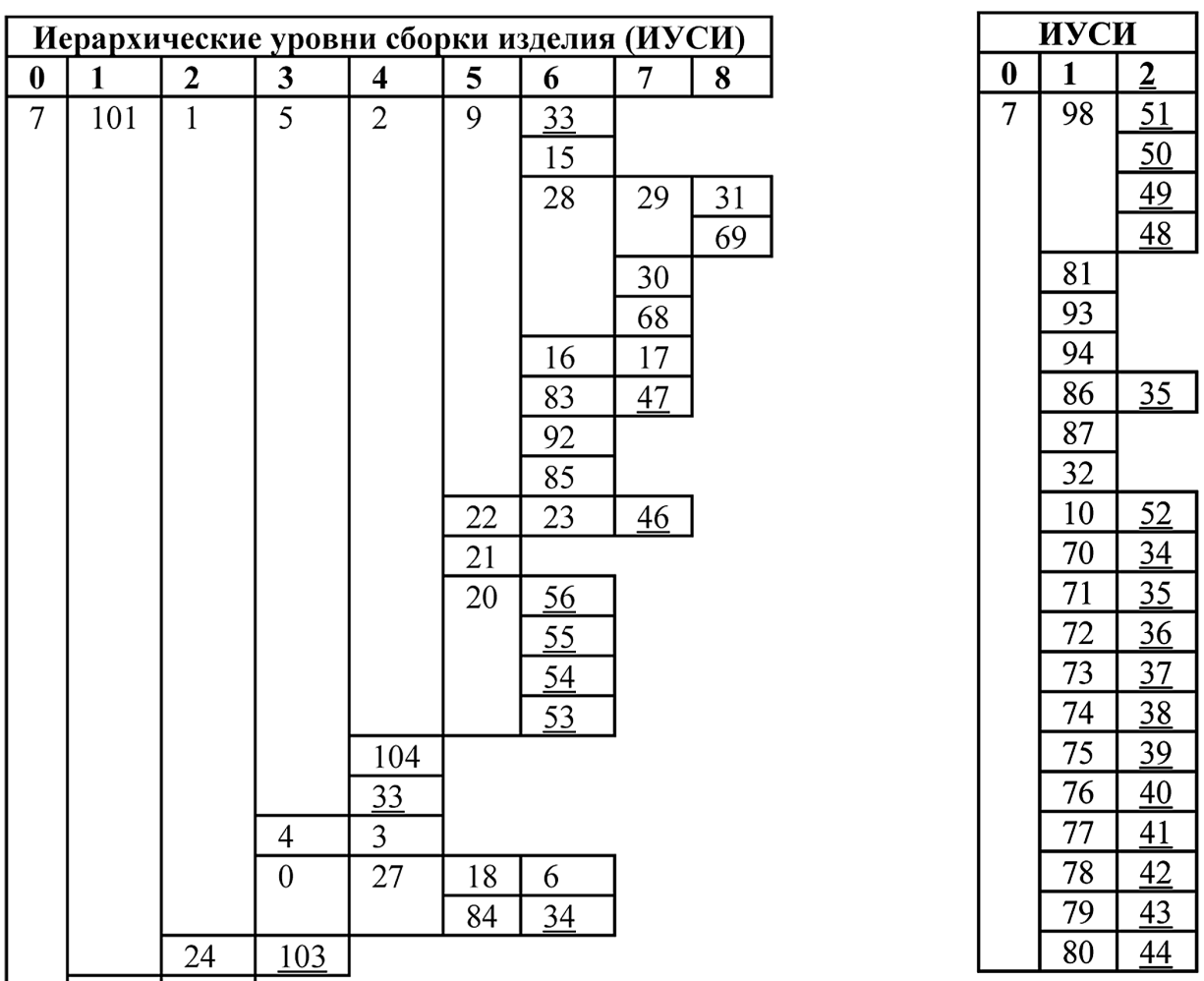

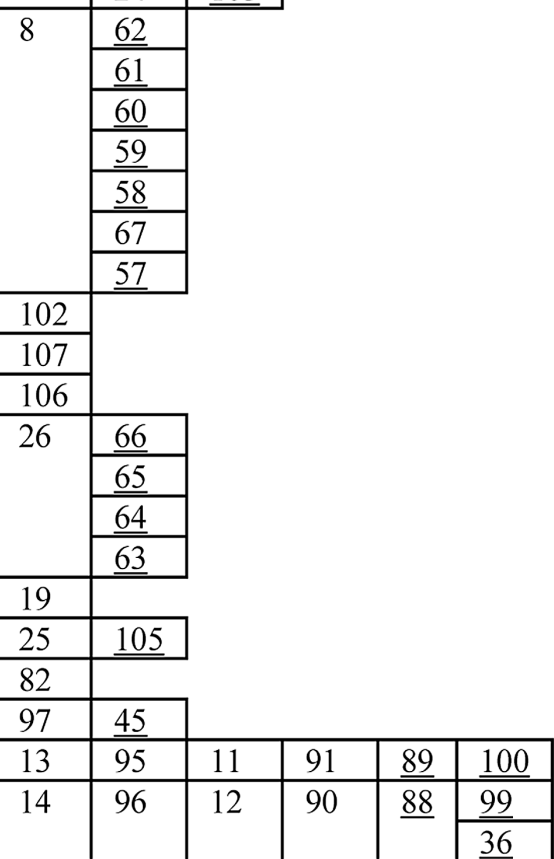

Общие выводы

1. Обеспечение стабильного качества сборки высокоточных изделий в условиях мелкосерийного производства может быть реализовано на основе управления процессами сборки и механической обработки деталей узла на основе их синхронизации.
2. Для реализации такого подхода процесс сборки изделия должен представлять собой систему, в которой формируется иерархия деталей, собираемых в узел на основе их конструктивных и технологических признаков. Для чего создается виртуальный сборочный узел, в котором присутствуют как реальные размеры уже обработанных деталей, так и 
предполагаемые размеры деталей по их чертежам до обработки.

3. Механическая обработка деталей должна быть синхронизирована с их сборкой в узле, при этом контроль качества сборки узла по конструкторским размерным цепям должен быть тем механизмом, который управляет процессами финишной обработки поверхностей деталей и, в случае необходимости, выполняет их переналадку.

4. При таком подходе гарантируется 100\% собираемость узла, так как контроль механической обработки контактирующих поверхностей деталей превращается в контроль собираемости узла по результатам расчета замыкающих звеньев конструкторских размерных цепей. При этом можно оперативно влиять на процесс сборки путем изменения размеров реальных деталей в виртуальном сборочном узле.

\section{Литература}

1. Кравченко Л.С. Розмірний аналіз при проектуванні, виготовленні й складанні [Текст]: навч. посібник [для студ. вищ. навч. закл.] / Л.С. Кравченко. Харків: НТУ «ХПІ», 2009. - 356с.

2. Шелковий О. М., Мартинов М. С., Набока О. В. Дослідження продуктивності і надійності складання вузла «Гідроагрегат» в умовах автоматизованого виробництва // Вісник Національного технічного університету «Харківський політехнічний інститут». Збірник наукових праць. Серія: Технології в машинобудуванні. - Харків: НТУ «ХПІ». - 2015. № 40(1149). - С. 108-112.

3. Шелковий О.М., Фадєєв В.А., Набока О.В. Підвищення ефективності зборки складних машинобудівних виробів на засадах інтеграції механоскладальних операцій //Високі технології в машинобудуванні: зб. наук, праць / Під заг. ред. проф. О.М. Шелкового, редкол.: проф. І.М. Пижов (голова) та ін. - Харків, НТУ "ХПІ", 2018. - Вип. 1 (28). - С.192 - 205.

4. Шелковой А., Клочко А., Набока Е. Имитационное моделирование в задачах механосборочного производства /Авторы: Шелковой А., Клочко А., Набока E. - Saarbrbcken, Germany: LAP LAMBERT Academic Publishing, 2015. - 528 c.

Shelkovoj A. N., Naboka E. V.

National Technical University «Kharkiv Polytechnic Institute». Ukraine, Kharkiv

\section{INTEGRATION OF AUTOMATED MECHANICAL ASSEMBLY PRODUCTION OF HIGH-PRECISION ENGINEERING PRODUCTS AS AN ALTERNATIVE TO THEIR SELECTIVE ASSEMBLY}

The article analyzes the conditions for the assembly of high-precision products without fitting and selective selection. The initial premises of mechanical assembly based on the integration of production processes are formulated. The parameters of equipment control for assembly and processing of parts are highlighted. A mathematical model of the process of virtual assembly of a product and machining by cutting the parts included in it is presented, which allows assembling high-precision products without fitting and selective selection. [dx.doi.org/10.29010/087.4]

Keywords: high-precision product; virtual assembly; machining; design dimensional chain; technological dimensional chain; processing control; assembly control; simulation modeling; mathematical model.

\section{References}

[1] Kravchenko L.S. Rozmirnij analiz pri proektuvanni, vigotovlenni j skladanni [Text]: navch. posibnik [dlja stud. vishh. navch. zakl.] / L.S. Kravchenko. - Harkiv: NTU «HPI», 2009. - 356 p.

[2] Shelkovij O. M., Martinov M. S., Naboka O. V. Doslidzhennja produktivnosti i nadijnosti skladannja vuzla «Gidroagregat» v umovah avtomatizovanogo virobnictva // Visnik Nacional'nogo tehnichnogo universitetu «Harkivs'kij politehnichnij institut». Zbirnik naukovih prac. Serija: Tehnologiji v mashinobuduvanni. - Harkiv: NTU «HPI». - 2015. - № 40(1149). - Pp. 108-112.

[3] Shelkovij O.M., Fadejev V. A., Naboka O. V. Pidvishhennja efektivnosti zborki skladnih mashinobudivnih virobiv na zasadah integracii mehanoskladalnih operacij //Visoki tehnologiï v mashinobuduvanni: zb. nauk, prac' / Pid zag. red. prof. O.M. Shelkovogo, redkol.: prof. I.M. Pizhov (golova) ta in. - -Harkiv, NTU "HPI", 2018. - Vip. 1 (28). - Pp. 192 - 205.

[4] Shelkovoj A., Klochko A., Naboka E. Imitacionnoe modelirovanie v zadachah mehanosborochnogo proizvodstva /Avtory: Shelkovoj A., Klochko A., Naboka E. - Saarbrbcken, Germany: LAP LAMBERT Academic Publishing, 2015. - 528 p. 